\title{
Dos propuestas lúdicas y educativas, para aprender del barrio del Cabanyal
}

Silvia Molinero Domingo lacacerolaabollada@gmail.com

Mixuro Estudio de Arquitectura, España

mixuro@mixuro.com

Reference

Domingo, Silvia Molinero; (2012) "Dos propuestas lúdicas y educativas, para aprender del barrio del Cabanyal", p. 342-346. In: Barbosa, Helena; Quental, Joana [Eds]. Proceedings of the 2nd International Conference of Art, Illustration and Visual Culture in Infant and Primary Education. São Paulo: Blucher, 2015.

ISSN 2318-695X, ISBN: 978-989-98185-0-7

DOI 10.5151/edupro-aivcipe-65

Resumen

Este proyecto pedagógico consiste en el diseño y elaboración de una baraja compuesta de 32 cartas, con su correspondiente caja contenedora y un recortable basados en el patrimonio arquitectónico del Cabanyal, un barrio de la ciudad de Valencia, España. Son dos proyectos destinados a niñas y niños de entre 5 y 12 años, con el objetivo de aprender de un modo lúdico el patrimonio de un barrio, cuyos edificios están siendo amenazados. Esta propuesta creativa está enmarcada dentro del proyecto Cabanyal Archivo Vivo, organizado por La Esfera Azul y ha contado con el apoyo del Ministerio de Cultura español. Juego, arquitectura, patrimonio, recortable, baraja.

Abstract

This educational project is about designing and producing a 32 card shuffle, including its container card box, and a paper cutout both based on Cabanyal's architectural heritage, a neighborhood in the city of Valencia, Spain. These re two projects for children from 5 to 12 years old, with the goal of learning in a playful way the heritage of this particular district in which several buildings are threatened. This artistic proposal is enshrined within de Cabanyal Archivo Vivo project (Cabanyal live archive), organized by La Esfera Azul and it has been supported by the Spanish Ministry of Culture.

Keywords

Game, architecture, heritage, cutout, shuffle.

\section{El Cabanyal-Cañamelar}

El barrio del Cabanyal-Cañamelar situado al este de la ciudad de Valencia, perteneciente al distrito de Poblados Marítimos, es un barrio marinero que ofrece numerosas características y propiedades singulares. Su peculiar trama en retícula deriva de las alineaciones de las antiguas barracas paralelas

al mar. Pueblo principalmente de pescadores, pronto se convirtió en una zona de interés como lugar de descanso y ocio. Existen numerosos ejemplos de arquitectura singular como los miramares (torres sobre viviendas desde los que observar la llegada de los barcos pesqueros) o fachadas de estilo modernista-popular. Este barrio, protegido desde 1988, fue declarado Bien de Interés Cultural en 1993. 
and International Conference Art, Illustration and Visual Culture in Infant and Primary Education
${ }^{\circ}$ Congreso Internaciona|

Arte, llustración y Cultura Visual en Educación Infantil y Primaria
Congresso Internacional

de Arte, Uustração e Cultura Visual

na Educação Infantil e Primária

\section{Plataformas, asociaciones, vecinos en oposición y propuestas creativas contra la desaparición de las peculiaridades del barrio.}

\section{Cabanyal Archivo Vivo}

Existe un proyecto de interés municipal de prolongar una gran avenida existente atravesando el trazado del barrio, con el objetivo de alcanzar el mar. Pero este proyecto ha encontrado un fuerte rechazo, principalmente por los vecinos afectados, pero también por diversos colectivos de la ciudad como la Asociación de Vecinos del Cabanyal Canyamelar o la Plataforma Salvem el Cabanyal que desde su creación, hace 14 años, llevan adoptando una serie de medidas legales a favor de la rehabilitación, para evitar la desaparición de 1651 viviendas y seccionar esta población en dos mitades, como pretende el plan del Ayuntamiento.

A su vez numerosas entidades y particulares, que se han adherido a la lucha por la protección de este conjunto histórico protegido, llevan proponiendo acciones creativas para dotar de visibilidad y dar a conocer el patrimonio del barrio. Una de estas iniciativas es Cabanyal Archivo Vivo un conjunto de trabajos artísticos desarrollados para el espacio real e internet, organizado por la Asociación La Esfera Azul con el apoyo del Ministerio de Cultura español, cuya intención es dotar de valor, identidad y memoria el patrimonio de este singular barrio valenciano. Las propuestas enmarcadas en esta iniciativa, se presentaron en el contexto de unas conferencias y mesas redondas celebradas en el mes de diciembre de 2011. También el trabajo que a continuación describiremos.

La invitación que recibimos para participar en Archivo vivo pedía que elaboráramos dos juegos, uno haciendo un juego de mesa destinado a público infantil que enseñara el patrimonio del barrio del Cabanyal de Valencia y otro retomando los recortables de la colección La cacerola abollada, una serie de recortables realizados en láminas A-3 que proponían recortar y montar algunas de las viviendas de interés arquitectónico para la IV Edición de Cabanyal Portes Obertes del año 2001. Portes Obertes es un evento de arte contemporáneo en el que los vecinos del barrio ceden sus casas para mostrar los trabajos realizados por artistas. Una excusa para que el público que visita este evento pueda conocer de primera mano las características singulares de las casas amenazadas por derribo, y lo que quizá es más importante, encontrarse con quienes las habitan. Esta actividad viene celebrándose desde el 1998 y actualmente se encuentra en su X edición.
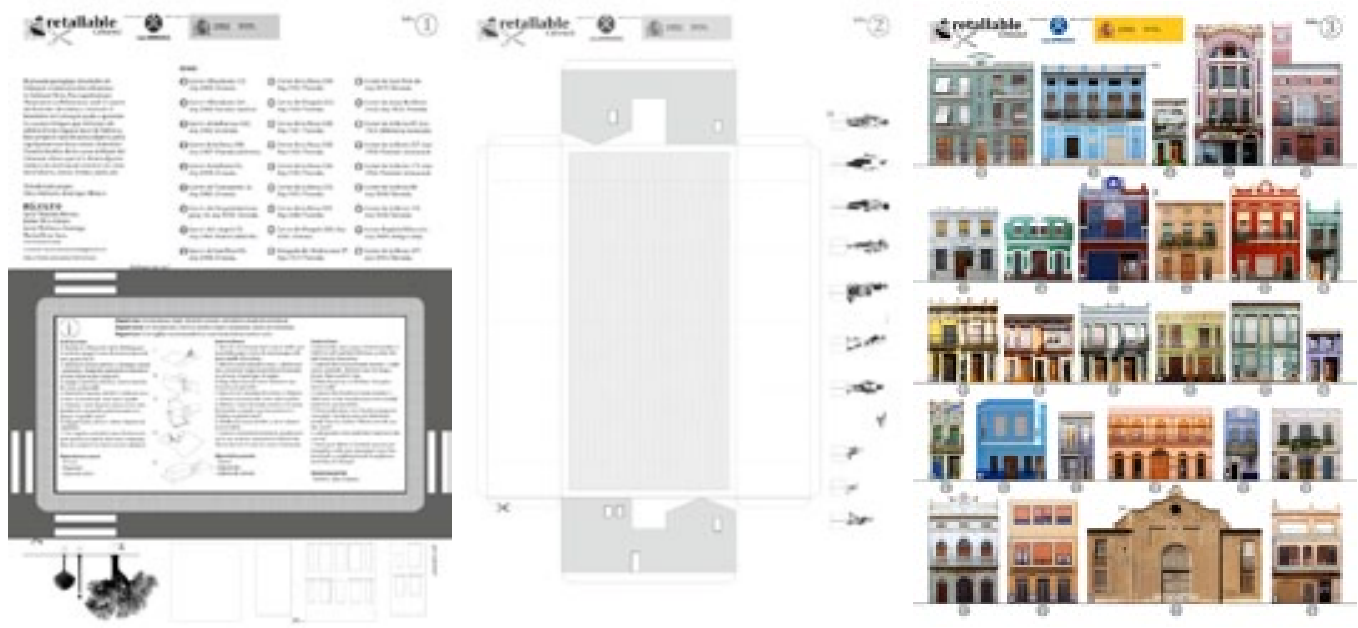
Art, Illustration and Visual Culture in Infant and Primary Education
Congreso Internacional

Arte, llustración y Cultura Visual en Educación Infantil y Primaria de Arte, llustração e Cultura Visual

na Educação Infantil e Primária
Figuras 4 y 5 . A la izquierda: niños y niñas de $3^{\circ}$ ciclo de infantil (marzo de 2012). Colegio Público Cervantes, Valencia, España. Foto: Silvia Molinero Domingo. A la derecha: Uno de los trabajos resultantes. Colegio Público Cervantes, Valencia, España. Foto: Silvia Molinero Domingo.
Figuras 6 y 7. A la izquierda: Algunas de las cartas de la baraja. Foto de Silvia Molinero Domingo (2011). A la derecha: Niñas y niños jugando con las cartas. Foto de Javier Molinero Domingo (2011).
Tras varias pruebas, el proceso creativo nos llevó a diseñar un recortable que añadiera, a la propuesta que realizamos en 2001, la posibilidad de construir una manzana típica del Cabanyal. La colección anterior proponía construir una vivienda y esta vez quisimos potenciar uno de los valores que posee este barrio, su configuración, por lo que el recortable te permite construir un modelo de manzana y si se plantea la actividad en el aula de un centro escolar, la posibilidad incluso de construir un barrio.

Recortable Cabanyal son 3 láminas de tamaño A-3. La lámina número 1 incluye la base sobre la que construir la manzana, el listado de 27 edificios emblemáticos del barrio junto a sus descripciones principales y algunas fachadas en blanco para que las niñas y niños a partir de 5 años, destinatarios principales de este juego, puedan pintar libremente y añadir sus propias creaciones. La hoja número 2 incluye el prisma sobre el que añadir las fachadas de las casas y la lámina 3 contiene las fotografías a color de las fachadas de las 27 viviendas.
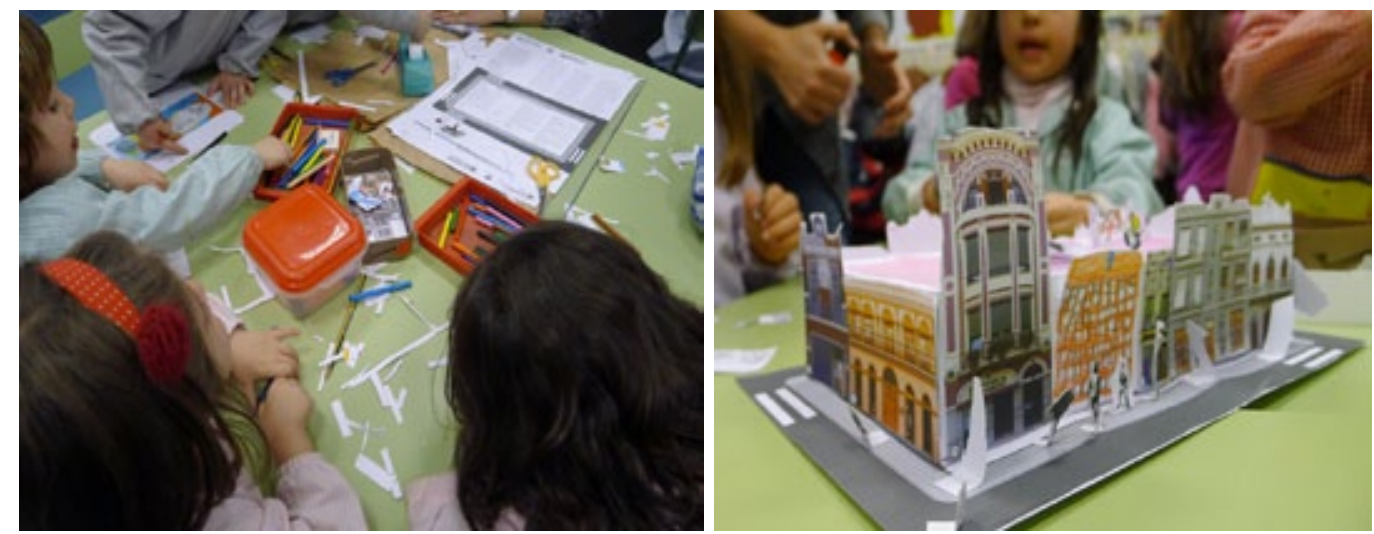

Con respecto a la segunda propuesta, quisimos diseñar un juego con el que se sintieran familiarizados actualmente y observándolos coleccionando e intercambiando cromos a la salida del colegio, pensamos en una baraja cuyas cartas estuvieran protagonizadas por algunos edificios del Cabanyal. Baraja el Cabanyal se basa en aquellas cartas temáticas de hace algunos años sobre coches, aviones, motos o superhéroes cuyas características descritas en la propia carta te ayudaban a ganar la partida. En el caso de los vehículos se describían los kilómetros hora, la cilindrada o el tipo de motor y en el caso de los superhéroes la velocidad, la altura, el peso, la fuerza o las peleas ganadas. En nuestro caso el año de construcción, la superficie construida, el número de plantas y la distancia hasta alcanzar el mar, son las características que se describen en las 32 cartas que conforman la baraja.
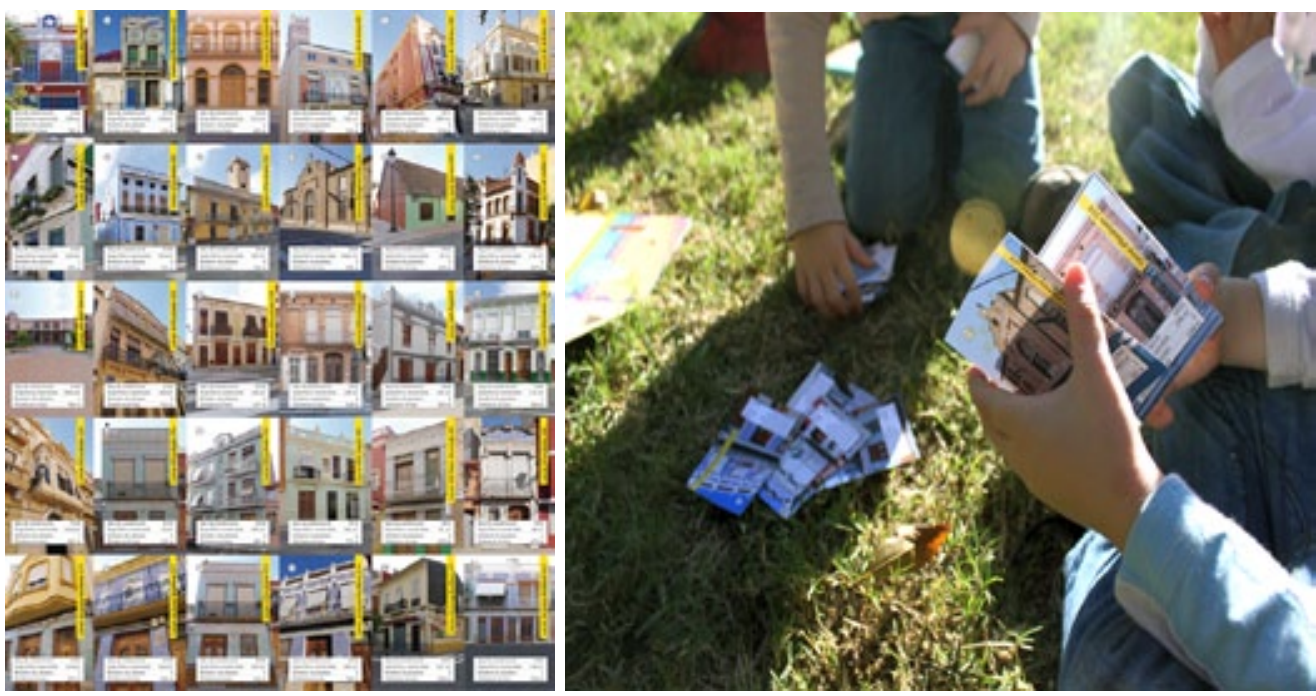
$2^{\text {nd }}$ International Conference Art, Illustration and Visual Culture in Infant and Primary Education $2^{\circ}$ Congreso Internaciona

Arte, Ilustración y Cultura Visual en Educación Infantil y Primaria
${ }^{\circ}$ Congresso Internacional

de Arte, Ilustração e Cultura Visual

na Educação Infantil e Primária

El principal objetivo del juego es ganar todas las cartas posibles. Las instrucciones te indican que se distribuyan todas las cartas entre los jugadores y se coloquen en montón boca abajo. El jugador "mano" observará las características de su vivienda, eligiendo aquella que le parezca más oportuna y la dirá en voz alta. El que tenga el valor mayor entre superficie construida o número de plantas, y el valor menor de año de construcción o distancia al mar, escogidas por el "mano", será el ganador y cogerá las cartas de esa baza. El ganador será "mano" en la siguiente jugada y así continúa el juego.

Gracias a la ayuda recibida del Ministerio de Cultura, se ha realizado una tirada de 1.000 ejemplares, poniendo a disposición sin coste alguno este material a todos los que conforman la comunidad escolar (equipo directivo, docentes, madres y padres) que tuvieran interés en desarrollar los juegos dentro del aula. La difusión de este trabajo y el interés suscitado ha hecho posible que esta actividad se haya llevado a cabo en diversos centros escolares de Madrid, Valencia, Barcelona, etc.

Previendo que todo el material impreso fuera distribuido, tanto la baraja como el recortable se ha adaptado a tamaño A-4, para el que lo desee, esté donde esté, pueda imprimírselo y Cada una de las bolsas de papel que se han entregado, contenía 7 hojas de las láminas 1, 2 y 3 del recortable, dos barajas y dos cajas para guardarlas. La propuesta invita a que formando grupos de 4 alumnas y alumnos se pueda jugar, mientras se aprende de este singular barrio de Valencia, formando pequeñas manzanas y juntarlas con el resto de compañeras y compañeros para formar un barrio.

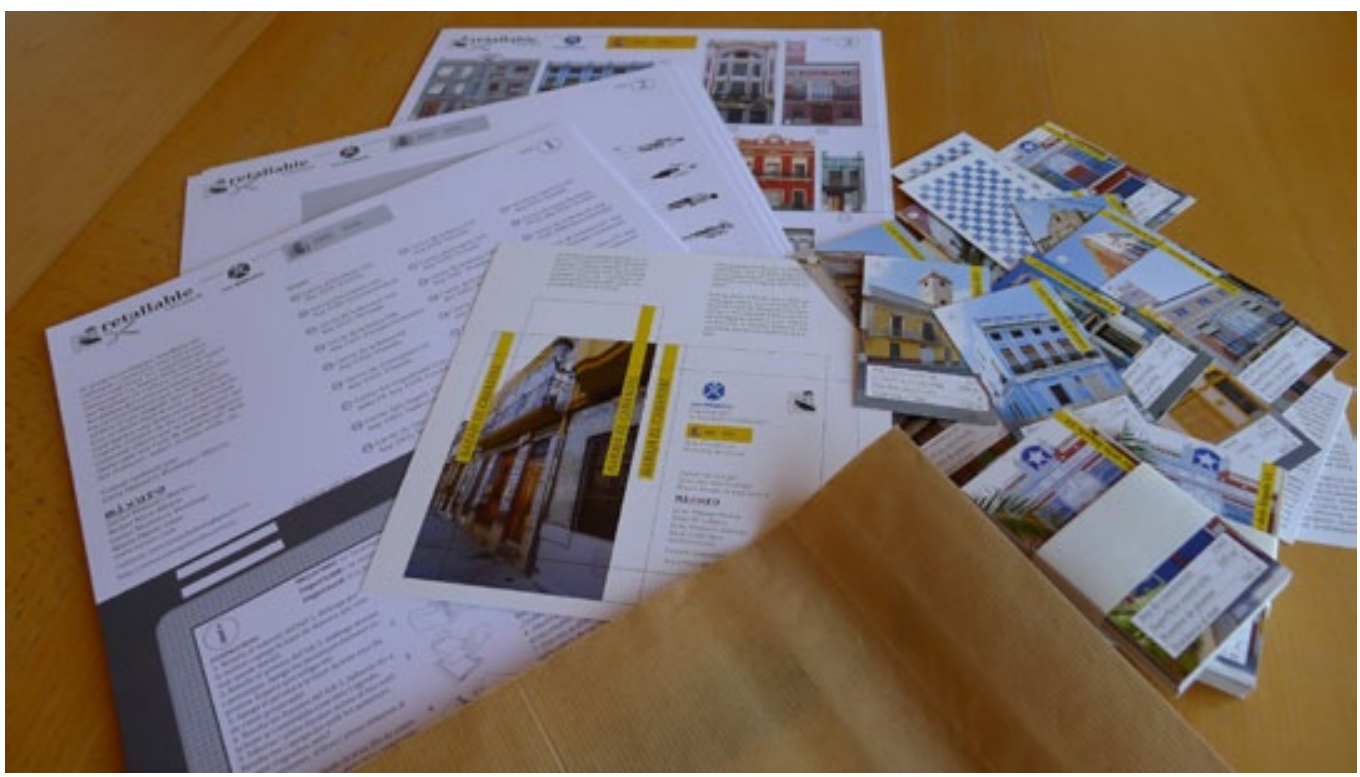

Previendo que todo el material impreso fuera distribuido, tanto la baraja como el recortable se ha adaptado a tamaño A-4, para el que lo desee, esté donde esté, pueda imprimírselo y hacerlo en casa. Puede descargar desde la página web de Cabanyal Arxiu Viu http://www. cabanyalarchivovivo.es.

Con estos dos proyectos lúdicos y educativos hemos querido, con una mirada curiosa como la de un niño, conocer y aprender de este barrio y a su vez enseñar a las generaciones más jóvenes el patrimonio que existe en esta ciudad. El Cabanyal ofrece numerosas características y propiedades singulares que a veces, por el mero hecho de tenerlas cercanas, pasan desapercibidas. Estos juegos ofrecen una manera de volver a mirar el barrio para el que ya lo conoce, o de conocerlo para el que todavía no lo ha visitado.

Los resultados que hemos conocido han sido considerablemente satisfactorios, los jóvenes han descubierto una nueva manera de aprender sobre la ciudad. Y los docentes lo han utilizado para enseñar a sus alumnas y alumnos qué es una manzana, un barrio, un patrimonio arquitectónico 
2nd International Conference Art, Illustration and Visual Culture in Infant and Primary Education

y el Cabanyal de Valencia. Un nuevo modo de aprender sobre las características que definen las viviendas de este singular barrio de Valencia.

La ilustración, el diseño y la metodología del juego nos han servido para mostrar la valía de un barrio, fomentando el pasar un buen rato enseñando el interés histórico de las casas de un barrio e informar a su vez de algunos datos relevantes como el año de construcción, el número de alturas, los metros cuadrados o la distancia de cada vivienda hasta alcanzar el mar.

Estos trabajos, con un gran componente visual y una metodología de la enseñanza lúdica, nos han ayudado a transmitir la puesta en valor de un barrio, cuyos edificios se están viendo amenazados. Tras su valoración con algunos docentes que han llevado a cabo la propuesta en sus aulas, todos podemos decir que Aprendiendo del Cabanyal ha favorecido considerablemente la transmisión de manera amena, rápida y concreta los conceptos planteados. Experimentar, crear, jugar, dibujar, colorear, divertirse, recortar y construir han sido utilizados con un nuevo enfoque educativo para afianzar conocimientos.

\section{Referencias bibliográficas}

VVAA, (2011). Cabanyal Archivo Vivo. Valencia, Archivo Vivo, 2011.

VVAA, (2007). IX Edició Cabanyal Portes Obertes-2007. Trobada d'escriptors pel Cabanyal, "El comprimís de l'escrptor". Valencia, Plataforma Salvem el Cabanyal, 2007.

VVAA, (2008). X Cabanyal Portes Obertes 08. Els primers 10 anys [1998-2008]. Art, resistencia i participació ciudadana. Valencia, Plataforma Salvem el Cabanyal, 2008.

VVAA, (2005). VIII Cabanyal Portes Obertes 2005 Art i ciutadania. Plataforma Salvem el CabanyalCanyamelar. Valencia, Plataforma Salvem el Cabanyal, 2005.

Ferrer, V. (2011). Benvinguts al Cabanyal. Valencia, Media vaca, 2011.

VVAA, (2009). Cases de El Cabanyal, maneres de viure: XI edició Cabanyal Portes Obertes. Valencia, Plataforma Salvem el Cabanyal-Canyamelar-Cap de França, 2009.

VVAA, (2015). VIII Cabanyal portes obertes 2005: art i ciutadania. València, Plataforma Salvem el Cabanyal, 2005

Martínez, E., (2010). Un Cabanyal de vinyetes: Cabanyal Portes Obertes 2010: XII edició, del 5 al 21 de novembre de 2010. Plataforma Salvem el Cabanyal-Canyamelar-Cap de França. Valencia, 2010.

Herrero, L. Varea, A, (2011). ¡Juégate el tipo!: arquitectura y vivienda para el barrio del Cabanyal. Valencia. Universidad Politécnica de Valencia, 2011.

<URL: http://www.cabanyalarchivovivo.es/ [Consult. 201205 28]

<URL: http://www.cabanyal.com/ [Consult. 201205 28] 